\title{
A Limit Theorem for Random Allocations
}

István Fazekas (Corresponding author)

Faculty of Informatics, University of Debrecen

PO box 12, Debrecen 4010, Hungary

E-mail: fazekasi@inf.unideb.hu

József Túri

Faculty of Mechanical Engineering, University of Miskolc

3515 Miskolc-Egyetemváros, Hungary

E-mail: TuriJ@abrg.uni-miskolc.hu

Received: November 14, 2011 Accepted: November 29, 2011 Published: February 1, 2012

doi:10.5539/jmr.v4n1p17 URL: http://dx.doi.org/10.5539/jmr.v4n1p17

\begin{abstract}
A limit theorem is presented for random allocations. For a fixed period we allocate $m$ balls into $N$ boxes. We repeat the experiment throughout $n$ periods. Let $p_{q}$ denote the probability that we do not place more than $q$ balls into any of the $N$ boxes during any of the $n$ repetitions. The limit of $p_{q}$ is determined when $m, n, N \rightarrow \infty$.
\end{abstract}

Keywords: Limit theorem, Random allocation

2010 Mathematics subject classification: 60C05, 60F05

\section{Introduction and Main Result}

Let balls be placed successively and independently into $N$ boxes. At each allocation the ball can fall into each box with probability $1 / N$. During a fixed period (for a day, say) we allocate $m$ balls. We repeat the experiment throughout $n$ days. Let $p_{q}$ denote the probability that we do not place more than $q$ balls into any of the $N$ boxes during any of the $n$ days.

(Avkhadiev \& Chuprunov, 2007) proved the following

Theorem A (Avkhadiev \& Chuprunov, 2007, Theorem 2) Let $m \geq 2$. Then

$$
p_{1}=\left(1-\frac{1}{N}\right)^{n}\left(1-\frac{2}{N}\right)^{n} \ldots\left(1-\frac{m-1}{N}\right)^{n} .
$$

If $m$ is fixed and $n, N \rightarrow \infty$ such that $n / N \rightarrow \alpha$, then $p_{1} \rightarrow e^{-\frac{m(m-1)}{2} \alpha}$; if $2 \leq q \leq m-1$, then $p_{q} \rightarrow 1$ as $n, N \rightarrow \infty$ such that $n / N \leq \alpha^{\prime}<\infty$.

We extend the above theorem in the following sense: To obtain non-trivial limit for $p_{q}$ when $q>1$ we have to consider growing number of balls. We expect that the rate of convergence of $m, n, N$ to $\infty$ will determine some $q$ such that lim $p_{q}$ is non-trivial, but $\lim p_{q-1}=0$ and $\lim p_{q+1}=1$. Our main result is the following

Theorem 1 Let $q$ be a fixed positive integer. Assume that $m, n, N \rightarrow \infty$ such that

$$
\frac{n}{N^{q}}\left(\begin{array}{c}
m \\
q+1
\end{array}\right) \rightarrow \alpha
$$

where $\alpha$ is a positive finite number and

$$
\frac{m^{2}}{N} \rightarrow 0
$$

Then

$$
\lim p_{l}= \begin{cases}0 & \text { if } 0 \leq l<q, \\ e^{-\alpha} & \text { if } l=q, \\ 1 & \text { if } l>q .\end{cases}
$$




\section{Remark 1}

$$
\left(1-\frac{1}{N^{l}}\left(\begin{array}{c}
m \\
l+1
\end{array}\right)\right)^{n} \leq p_{l} \leq\left(1-\frac{1}{N^{l}}\left(\begin{array}{c}
m \\
l+1
\end{array}\right)(1-\varepsilon)\right)^{n}
$$

for $l=1,2, \ldots, m-1$, where $\varepsilon>0$ and $\varepsilon \rightarrow 0$ if $m \rightarrow \infty$ and $N \rightarrow \infty$ such that $m^{2} / N \rightarrow 0$.

We want to mention that random allocations have been widely studied. See the classic papers (Weiss, 1958; Rényi, 1962) and (Békéssy, 1963), the traditional monograph (Kolchin, Sevast'yanov \& Chistyakov, 1978). For more recent results, the reader can consult (Timashev, 2000) and (Chuprunov \& Fazekas, 2005).

\section{Proof of Main Result}

The proof is based on the following

Theorem B (Avkhadiev \& Chuprunov, 2007, Theorem 1) Let $m \geq 2$ and $1 \leq q \leq m-1$. Then

$$
p_{q}=\left(1-\frac{A_{q}}{q ! N}\right)^{n}
$$

where

$$
A_{q}=\left.\sum_{l=q}^{m-1} \frac{1}{N^{l-1}} \frac{d^{l}\left[z^{q}\left(f_{q}(z)\right)^{N-1}\right]}{d z^{l}}\right|_{z=0},
$$

and

for any non-negative integer $k$.

$$
f_{k}(z)=1+\frac{z}{1 !}+\frac{z^{2}}{2 !}+\cdots+\frac{z^{k}}{k !}
$$

We mention that the proof of Theorem B is based on a result by (Timashev, 2000).

Remark 2 First we consider the case $q=1$ because it is very simple and shows that Condition (2) is natural. Effectively, in virtue of (1),

$$
\ln p_{1}=n \sum_{i=0}^{m-1} \ln \left(1-\frac{i}{N}\right)
$$

Using Taylor's expansion $\ln (1-x)=-x-x^{2} /\left(2(1-\vartheta x)^{2}\right)$ with $\vartheta \in(0,1)$, we obtain

$$
\ln p_{1}=-n \sum_{i=0}^{m-1} \frac{i}{N}-\frac{n}{2} \sum_{i=0}^{m-1}\left(\frac{i}{N}\right)^{2}\left(1-\vartheta_{i} \frac{i}{N}\right)^{-2} .
$$

For the first addend in (8) we have $-n \sum_{i=0}^{m-1} \frac{i}{N}=-\frac{n}{N}\left(\begin{array}{c}m \\ 2\end{array}\right) \rightarrow-\alpha$. Assuming $m<N$, the second addend in (8) can be handled as

$$
\begin{gathered}
\left|-\frac{n}{2} \sum_{i=0}^{m-1}\left(\frac{i}{N}\right)^{2}\left(1-\vartheta_{i} \frac{i}{N}\right)^{-2}\right| \leq \frac{n}{2 N^{2}}\left(1-\frac{m}{N}\right)^{-2} \sum_{i=0}^{m-1} i^{2} \\
\leq \frac{n}{2 N^{2}}\left(1-\frac{m}{N}\right)^{-2} \frac{(m-1) m(2 m-1)}{6}=\frac{n}{N}\left(\begin{array}{c}
m \\
2
\end{array}\right) \frac{2 m-1}{6 N}\left(1-\frac{m}{N}\right)^{-2} \rightarrow 0 .
\end{gathered}
$$

Therefore $p_{1} \rightarrow e^{-\alpha}$, if $\frac{n}{N}\left(\begin{array}{c}m \\ 2\end{array}\right) \rightarrow \alpha$.

Proof of Theorem 1 By the Leibniz formula, we have for $v \geq q$

$$
\left.\frac{d^{\nu}\left[z^{q}\left(f_{q}(z)\right)^{N-1}\right]}{d z^{v}}\right|_{z=0}=\left.\left.\sum_{k=0}^{v}\left(\begin{array}{l}
v \\
k
\end{array}\right) \frac{d^{k}\left[z^{q}\right]}{d z^{k}}\right|_{z=0} \frac{d^{\nu-k}\left[\left(f_{q}(z)\right)^{N-1}\right]}{d z^{v-k}}\right|_{z=0}=\left.\left(\begin{array}{l}
v \\
q
\end{array}\right) q ! \frac{d^{v-q}\left[\left(f_{q}(z)\right)^{N-1}\right]}{d z^{v-q}}\right|_{z=0}
$$

Therefore

$$
A_{q}=\left.\sum_{l=q}^{m-1} \frac{1}{N^{l-1}}\left(\begin{array}{l}
l \\
q
\end{array}\right) q ! \frac{d^{l-q}\left[\left(f_{q}(z)\right)^{N-1}\right]}{d z^{l-q}}\right|_{z=0}
$$

We see that $\frac{d^{l}\left(f_{k}(z)\right)}{d z^{l}}=f_{k-l}(z)$, where $f_{h}(z)$ is defined as 0 for $h<0$. We have $\left.\left(f_{q}(z)\right)^{t}\right|_{z=0}=1=t^{0}$ for $q \geq 0$. Now we shall show that for $t \geq k \geq 1$ and $q \geq 1$

$$
t_{(k)} \leq\left.\frac{d^{k}\left[\left(f_{q}(z)\right)^{t}\right]}{d z^{k}}\right|_{z=0} \leq t^{k}
$$


where $t_{(k)}=t(t-1) \ldots(t-k+1)$. We will prove these inequalities by induction. For $k=1$ we have

$$
\left.\frac{d\left[\left(f_{q}(z)\right)^{t}\right]}{d z}\right|_{z=0}=\left.\left.t\left(f_{q}(z)\right)^{t-1}\right|_{z=0} f_{q}^{\prime}(z)\right|_{z=0}=\left.\left.t\left(f_{q}(z)\right)^{t-1}\right|_{z=0} f_{q-1}(z)\right|_{z=0}=t .
$$

By the Leibniz formula,

$$
\begin{gathered}
\left.\frac{d^{k+1}\left[\left(f_{q}(z)\right)^{t}\right]}{d z^{k+1}}\right|_{z=0}=\left.\frac{d^{k}\left[t\left(f_{q}(z)\right)^{t-1} f_{q-1}(z)\right]}{d z^{k}}\right|_{z=0} \\
=\left.\left.t \sum_{l=0}^{k}\left(\begin{array}{l}
k \\
l
\end{array}\right) \frac{d^{l}\left[\left(f_{q}(z)\right)^{t-1}\right]}{d z^{l}}\right|_{z=0} f_{q-1-(k-l)}(z)\right|_{z=0}=\left.t \sum_{l=k+1-q}^{k}\left(\begin{array}{l}
k \\
l
\end{array}\right) \frac{d^{l}\left[\left(f_{q}(z)\right)^{t-1}\right]}{d z^{l}}\right|_{z=0}=F .
\end{gathered}
$$

Using the induction hypothesis,

$$
F \geq t \sum_{l=k+1-q}^{k}\left(\begin{array}{l}
k \\
l
\end{array}\right)(t-1)_{(l)} \geq t(t-1)_{(k)}=(t)_{(k+1)}
$$

and

$$
F \leq t \sum_{l=k+1-q}^{k}\left(\begin{array}{l}
k \\
l
\end{array}\right)(t-1)^{l} \leq t t^{k}=t^{k+1}
$$

Hence (10) holds and can be used as follows

$$
\begin{aligned}
\frac{A_{q}}{q ! N} \geq \sum_{k=q}^{m-1} \frac{1}{N^{k}}\left(\begin{array}{l}
k \\
q
\end{array}\right)(N-1)_{(k-q)}=\frac{1}{N^{q}} \sum_{k=q}^{m-1}\left(\begin{array}{l}
k \\
q
\end{array}\right) \frac{(N-1)(N-2) \cdots(N-(k-q))}{N^{k-q}} \geq \\
\geq \frac{1}{N^{q}} \sum_{k=q}^{m-1}\left(\begin{array}{l}
k \\
q
\end{array}\right)(1-\varepsilon)=\frac{1}{N^{q}}\left(\begin{array}{c}
m \\
q+1
\end{array}\right)(1-\varepsilon)
\end{aligned}
$$

where $\varepsilon>0$ and $\varepsilon \rightarrow 0$ as $m, N \rightarrow \infty$ such that Condition (3) is satisfied. In (11), we only need prove $(N-1)(N-2) \cdots(N-(k-q)) / N^{k-q} \geq 1-\varepsilon$. To do so, given $k=q, q+1, \ldots, m-1$ we consider

$$
\begin{gathered}
0 \geq \ln \left(\frac{(N-1)(N-2) \cdots(N-(k-q))}{N^{k-q}}\right) \geq \sum_{l=1}^{m-1-q} \ln \frac{N-l}{N} \geq \\
\geq \int_{1}^{m-1-q} \ln \frac{N-x}{N} d x=\left[(a-N) \ln \left(1-\frac{a}{N}\right)-a\right]-\left[(1-N) \ln \left(1-\frac{1}{N}\right)-1\right]
\end{gathered}
$$

where $a=m-1-q$. Note that the second addend in the previous expression tends trivially to 0 ; whereas the first addend shows the same tendency due to Condition (3) and Taylor's expansion for $\ln (1-a / N)$. This involves that (11) holds.

Again by (10),

$$
\begin{gathered}
\frac{A_{q}}{q ! N} \leq \sum_{k=q}^{m-1} \frac{1}{N^{k}}\left(\begin{array}{l}
k \\
q
\end{array}\right)(N-1)^{k-q}=\frac{1}{N^{q}} \sum_{k=q}^{m-1}\left(\begin{array}{l}
k \\
q
\end{array}\right)\left(\frac{N-1}{N}\right)^{k-q} \\
\leq \frac{1}{N^{q}} \sum_{k=q}^{m-1}\left(\begin{array}{l}
k \\
q
\end{array}\right)=\frac{1}{N^{q}}\left(\begin{array}{c}
m \\
q+1
\end{array}\right) .
\end{gathered}
$$

Thus the upper and the lower bounds of $\frac{A_{q}}{q ! N}$ are of the form $\frac{1}{N^{q}}\left(\begin{array}{c}m \\ q+1\end{array}\right)$ and $\frac{1}{N^{q}}\left(\begin{array}{c}m \\ q+1\end{array}\right)(1-\varepsilon)$, respectively, where $\varepsilon>0$ and $\varepsilon \rightarrow 0$ as $m, N \rightarrow \infty$ such that Condition (3) is satisfied. In virtue of (6),

$$
\left(1-\frac{1}{N^{q}}\left(\begin{array}{c}
m \\
q+1
\end{array}\right)\right)^{n} \leq p_{q}=\left(1-\frac{A_{q}}{q ! N}\right)^{n} \leq\left(1-\frac{1}{N^{q}}\left(\begin{array}{c}
m \\
q+1
\end{array}\right)(1-\varepsilon)\right)^{n}
$$

where $\varepsilon>0$ and $\varepsilon \rightarrow 0$ as $m, N \rightarrow \infty$ such that Condition (3) is satisfied. Consequently, in virtue of (2),

$$
p_{q} \rightarrow e^{-\alpha} \text {. }
$$

Above we applied only Condition (3) (and we did not apply Condition (2)) to obtain (14). Consequently we have proved Remark 1. 
Now consider $p_{q+1}$ and $p_{q-1}$. Using (5),

$$
p_{q+1} \approx\left(1-\frac{\frac{n}{N^{q+1}}\left(\begin{array}{c}
m \\
q+2
\end{array}\right)}{n}\right)^{n} \rightarrow e^{0}=1,
$$

since $\frac{n}{N^{q+1}}\left(\begin{array}{c}m \\ q+2\end{array}\right) \rightarrow 0$ in virtue of (2) and (3). (Here $a_{s} \approx b_{s}$ means that $a_{s}-b_{s} \rightarrow 0$ as $s \rightarrow \infty$ ). Moreover,

$$
p_{q-1} \approx\left(1-\frac{\frac{n}{N^{q-1}(}\left(\begin{array}{c}
m \\
q
\end{array}\right)}{n}\right)^{n} \rightarrow e^{-\infty}=0
$$

because $\frac{n}{N^{q-1}}\left(\begin{array}{l}m \\ q\end{array}\right) \rightarrow \infty$ and $\frac{n}{N^{q-1}}\left(\begin{array}{c}m \\ q\end{array}\right) / n \rightarrow 0$ by (2) and (3).

\section{Acknowledgment}

The authors are indebted to A. Chuprunov for helpful comments. The authors would like to thank the referee for useful remarks which helped to clarify the exposition.

\section{References}

Avkhadiev, F. G., \& Chuprunov, A. N. (2007). The probability of a successful allocation of ball groups by boxes. Lobachevskii J. Math, 25, 3-7.

Békéssy, A. (1963). On classical occupancy problems. I. Magyar Tud. Akad. Mat. Kutató Int. Közl, 8 (1-2), 59-71.

Chuprunov, A., \& Fazekas, I. (2005). Inequalities and strong laws of large numbers for random allocations. Acta Math. Hungar, 109 (1-2), 163-182.

Kolchin, V. F., Sevast'yanov, B. A., \& Chistyakov, V. P. (1978). Random allocations. V. H. Winston \& Sons, Washington D. C.

Rényi, A. (1962). Three new proofs and a generalization of a theorem of Irving Weiss. Magyar Tud. Akad. Mat. Kutató Int. Közl, 7 (1-2), 203-214.

Timashev, A. N. (2000). On the asymptotics of large deviations in schemes for allocating particles to different cells of bounded sizes. Teor. Veroyatnost. i Primenen. 45 (3), 521-535 (in Russian). Translation into English in Theory Probab. Appl, 45 (3), 494-506 (2002).

Weiss, I. (1958). Limiting distributions in some occupancy problems. Ann. Math. Statist, 29 (3), 878-884. 